\title{
Association between neutrophil to lymphocyte ratio and mortality among community acquired pneumonia patients: a meta-analysis
}

\author{
Osama Alzoubi, Asim Khanfar \\ School of Medicine, University of Jordan, Amman, Jordan
}

\begin{abstract}
The neutrophil to lymphocyte ratio (NLR) is an emerging biomarker used in the prognosis of many conditions. We aimed to conduct a meta-analysis to assess the prognostic accuracy of the
\end{abstract}

Correspondence: Osama Alzoubi, School of Medicine, University of Jordan, Taki Al Deenst. Amman 11953, Jordan. Tel. +962.791996046. E-mail: osama-alzoubi@hotmail.com

Key words: Neutrophil to lymphocyte ratio; community acquired pneumonia; marker; mortality.

Contributions: OA, AK, designed the study; carried out the literature search and extracted data, wrote and reviewed the manuscript; OA, analyzed the data. All the authors have read and approved the final version of the manuscript and agreed to be accountable for all aspects of the work.

Conflict of interest: The authors have no relevant affiliations or financial involvement with any organization or entity with a financial interest in or financial conflict with the subject matter or materials discussed in the manuscript. This includes employment, consultancies, honoraria, stock ownership or options, expert testimony, grants or patents received or pending, or royalties.

Ethics approval: No ethical approval will be needed because data from previous published studies in which informed consent was obtained will be extracted and analyzed.

Data Availability: All data extracted from studies and used in the analysis, in addition to the detailed analysis outcome is available in Supplementary File 2.

Acknowledgments: The authors thank the anonymous reviewers for their constructive comments.

Received for publication: 13 August 2021.

Accepted for publication: 16 November 2021.

Publisher's note: All claims expressed in this article are solely those of the authors and do not necessarily represent those of their affiliated organizations, or those of the publisher, the editors and the reviewers. Any product that may be evaluated in this article or claim that may be made by its manufacturer is not guaranteed or endorsed by the publisher.

${ }^{\circ}$ Copyright: the Author(s), 2021

Licensee PAGEPress, Italy

Monaldi Archives for Chest Disease 2022; 92:2050

doi: 10.4081/monaldi.2021.2050

This article is distributed under the terms of the Creative Commons Attribution-NonCommercial International License (CC BY-NC 4.0) which permits any noncommercial use, distribution, and reproduction in any medium, provided the original author(s) and source are credited.
NLR in determining mortality in patients with community acquired pneumonia (CAP). The PubMed, EBSCO, and Scopus databases were searched to find all relevant articles; 10 articles with 5220 patients were included. The pooled area under the curve (AUC) of NLR admission levels to predict 30-Day mortality of CAP patients was $0.706 ; 95 \%$ CI $(0.631$ to 0.781$)$, while the pooled AUC of NLR levels taken at 3-5 days was $0.882 ; 95 \% \mathrm{CI}$ (0.818 to 0.945$)$. Meta analysis also showed a significant difference in the NLR between the survivors and 30-day non-survivors. This difference was greater when NLR levels were taken at 3-5 days; standardized mean difference $(\mathrm{SMD})=1.646 ; 95 \% \mathrm{CI}$ (0.451 to 2.840) compared to NLR levels at admission SMD = $1.139 ; 95 \%$ CI ( 0.514 to 1.764$)$. These results show that the NLR has potential to be incorporated in the routine assessment and stratification of CAP patients, especially in the early-stage evolution (3-5 days), keeping in mind the availability and cost effectiveness of this test.

\section{Introduction}

Community acquired pneumonia (CAP) is a frequent and serious cause of morbidity and mortality worldwide [1,2] and causes significant economic burden on patients and healthcare systems $[3,4]$. Rapid assessment of the patient at the time of diagnosis is essential to determine the prognosis of the patient and adjust the management plan accordingly. Traditionally, two CAP severity predictors are used; CURB65 (confusion, urea, respiratory rate, blood pressure, and age 65 or older) [5] and the pneumonia severity index (PSI) [6], and both have been validated on large, diverse populations $[7,8]$. Various biomarkers have also been studied, including but not limited to $\mathrm{C}$-reactive protein (CRP), procalcitonin, copeptin, and proadrenomedullin [9-13]. These biomarkers have their own various limitations, and researchers are moving towards simpler tools such as the neutrophil-to-lymphocyte ratio (NLR).

The NLR has re-emerged as a predictor of severity of disease due to its availability and cost. It is a valuable tool that reflects the immune response. It has been studied in a variety of diseases, both acute and chronic [14-17]. De Jager et al. [18] was the first to study the prognostic accuracy of NLR in CAP patients, and showed that NLR has better prognostic accuracy than traditional inflammatory markers.

Even so, the use of NLR in determining the prognosis of CAP patients remains inconclusive. While many researchers such as Zhang et al. [19], Cataudella et al. [20] and others supported the use of NLR, others were more critical of its use. Avci and Perincek [21] reported that other biomarkers were more significant than NLR, and Lee et al. [22] specified that the day four of admission the NLR level is significant, not upon admission. Therefore, we 
conducted a meta-analysis to assess the prognostic accuracy of NLR in determining patient mortality.

\section{Materials and Methods}

\section{Search strategy and selection of studies}

We followed the preferred reporting items for systematic reviews and meta-analyses (PRISMA statement) guidelines to perform this meta-analysis [23]. It was prospectively registered on PROSPERO database (registration number: CRD42021260577). We systematically searched PubMed, Scopus and EBSCO databases for publications up to July1, 2021. The search terms were as follows: ("Neutrophil-lymphocyte" OR "Neutrophil-to-lymphocyte" OR "neutrophil lymphocyte" OR "neutrophil to lymphocyte" OR "NLR") and ("Community acquired pneumonia" OR "CAP"). The detail of search strategies used in different databases is shown in supplementary file. No language restrictions were imposed. To find additional citations, the reference lists of the included studies and recent review articles were screened when necessary.

The PICOS (population, intervention, comparator, outcome and study design) approach was utilized to define study eligibility. i) P: adult patients who died due to CAP; ii) I: assessment of admission NLR levels capacity to predict mortality in CAP patients; iii) C: adult patients who survived CAP; iv) O: the NLR value; v) S: observational studies including cross-sectional studies, cohort studies, case-control studies, or case series.

Two authors (OA and AK) independently screened all identified studies for inclusion in the final analysis. Any disagreement was resolved through discussion. Studies were selected if they met the following criteria: i) The study sample was community acquired pneumonia patients; ii) the study evaluated one of the following: a) The predictive capacity of NLR for 30-day mortality in CAP patients by reporting an AUC value, or b) The mean+-SD of NLR values in the survivors and non-survivor group; iii) the NLR values were taken at admission or at 3-5 days from admission. The exclusion criteria were as follows: i) case report, review, editorial, conference abstract, comment, letter, animal study; ii) insufficient data required for statistical analysis; iii) CAP patients who are pregnant or immunodeficient.

\section{Data extraction and quality assessment}

Two authors (AK and OA) independently extracted relevant information from individual studies, including first author, publication year, country, publication language, number of patients (male/female), mean age, area under curve (AUC) of NLR to predict mortality with related $95 \%$ confidence intervals and cut-off value, mean \pm SD NLR values and whether NLR level was taken at admission or at 3-5 days. The extracted information was double checked. The Newcastle-Ottowa Scale (NOS) was used to assess the quality of each paper according to three sections: selection, comparability, and exposure [24]. Papers can be graded as poor, fair, or good. AK and OA independently graded the quality of each paper, and any disagreement was resolved through discussion.

\section{Statistical analysis}

The meta-analysis of enrolled studies was performed using MedCalc ${ }^{\circledR}$ Statistical Software version 20 (MedCalc Software Ltd, Ostend, Belgium; https://www.medcalc.org; 2021). For patient data analysis, a random effects model was used and presented as a forest plot because the included articles had different characteristics. After data extraction has finished, studies were grouped according to outcome reported (AUC, mean NLR) then sub-grouped according to duration of mortality assessment (30-day mortality, in-hospital mortality, etc.) and time of NLR level taken (at admission, at 3-5 days). The standard error value of AUC was either obtained directly from the study or calculated from the $95 \%$ confidence interval, however if the study did not report the direct SE value or $95 \% \mathrm{CI}$ it was excluded from the AUC analysis. A summary AUC value regarding NLR ability to predict 30-day mortality in CAP patients was calculated under a random-effects model. Pooled and individual measures and their $95 \%$ confidence intervals $(95 \% \mathrm{CI})$ were illustrated in forest plots. The difference in the NLR between the non-survivors and survivors groups was assessed using the standardized mean difference (SMD). Publication bias was assessed by using a funnel plot and tested by using Egger's regression intercept test. Heterogeneity was quantified using the I 2 statistic, where I $>50 \%$ indicated significant heterogeneity between studies. Detailed output of the analysis is found in Supplementary File 2.

\section{Results}

\section{Literature search and study characteristics}

As a result of the literature search, a total of 724 studies were identified, including 128 from PubMed, 326 from Scopus and 270 from EBSCO. Figure 1 shows the study selection process through the PRISMA Flow diagram. In total, 258 duplicate publications were excluded. According to the inclusion and exclusion criteria, we excluded 363 studies by evaluating the titles and abstracts. The remaining 27 studies were further scrutinized by reading the full text. Finally, only 10 studies were included in this meta-analysis [18-21,25-30]; 6 reported the predictive AUC value on 30-day mortality [19,21,25-28] (of which 4 studies reported admission NLR levels, one study reported NLR at 3-5 days and 1 study reported both), 4 reported the mean NLR Values of survivors and 30 -day non-survivor groups $[19,20,25,26]$ (of which two reported admission NLR levels and two have reported both admission and 3-5 days NLR), and 3 reported the mean NLR values of survivors and in-hospital non-survivors groups $[18,29,30]$ (all of which only reported admission NLR levels). The characteristics of the included studies are shown in Table 1. Ten studies with 5220 patients were included; 5 studies were prospective and 5 were retrospective; 2 studies were carried out in Turkey, 4 in China, and the rest in Europe. The mean age of patients ranged between 57.1 and 80.3. All papers were considered to have good quality, and their NOS grades ranged from 6-8. The cutoff value of NLR predicting mortality used in three studies was 10 as put forward by Jager et al. [18]. Three other studies did not specify NLR cutoff value used.

\section{NLR and 30-day mortality}

Six studies have evaluated the predictive value of NLR and 30day mortality of CAP patients [19,21,25-28]. The pooled area under the curve (AUC) of NLR admission levels from five studies $[19,21,25,26,28]$ was $0.706 ; 95 \%$ CI $(0.631$ to 0.781$)$, while the pooled AUC of NLR levels taken at 3-5 days from two studies $[26,27]$ was $0.88295 \% \mathrm{CI}(0.818$ to 0.945$)$. Figure 2 shows the forest plot of the AUC meta-analysis. Four studies have evaluated the mean \pm SD NLR admission levels in the survivors and 30-day nonsurvivors $[19,20,25,26]$. Meta analysis of these studies showed a significant difference in the NLR between the survivors and 30day non-survivors $(\mathrm{SMD}=1.139,95 \%$ confidence interval $(\mathrm{CI})=$ 
0.514 to $1.764, \mathrm{p}<0.001)$. Two studies have evaluated the mean \pm SD NLR levels taken at 3-5 days in the survivors and 30-day nonsurvivors $[25,26]$. Meta analysis of these studies showed a significant difference in the NLR between the survivors and 30-day nonsurvivors $(\mathrm{SMD}=1.646,95 \%$ confidence interval $(\mathrm{CI})=0.451$ to $2.840, \mathrm{p}=0.007$ ) (Figure 3).

\section{NLR and in-hospital mortality}

Three studies have evaluated the mean +-SD NLR admission values in the survivors and In-Hospital non-survivors [18,29,30]. Meta-analysis of these studies showed a significant difference in the NLR between the survivors and in-hospital non-survivors $(\mathrm{SMD}=1.053,95 \%$ confidence interval $(\mathrm{CI})=0.813$ to 1.294 , $\mathrm{p}<0.001$ ) (Figure 3). Only one study was found to evaluate the predictive capacity of NLR and in-hospital mortality of CAP patients, accompanied with enough information to enable calculation of the standard error [29]. This has restricted us from reaching a pooled AUC value. No studies have evaluated the NLR levels taken at 35 days and in-hospital mortality.

\section{Assessment of publication bias and heterogeneity}

The funnel plot of the analysis of admission NLR predictive value and 30-day mortality (pooled AUC) did not reveal publication bias ( $p=0.2376$, Egger's test; Figure 4), while the heterogeneity was substantial $(\mathrm{I} 2=70.02 \%)$. The funnel plot of the analysis of differences between the mean admission NLR of the survivors and 30-day non-survivors did not reveal publication bias $(p=0.5230$, Egger's test; Figure 4$)$, while the heterogeneity was considerable ( $\mathrm{I} 2=89.13 \%)$. The funnel plot of the analysis of differences between the mean admission NLR of the survivors and inhospital non-survivors did not reveal publication bias $(\mathrm{p}=0.7409$, Egger's test; Figure 4), while the heterogeneity was substantial (I2

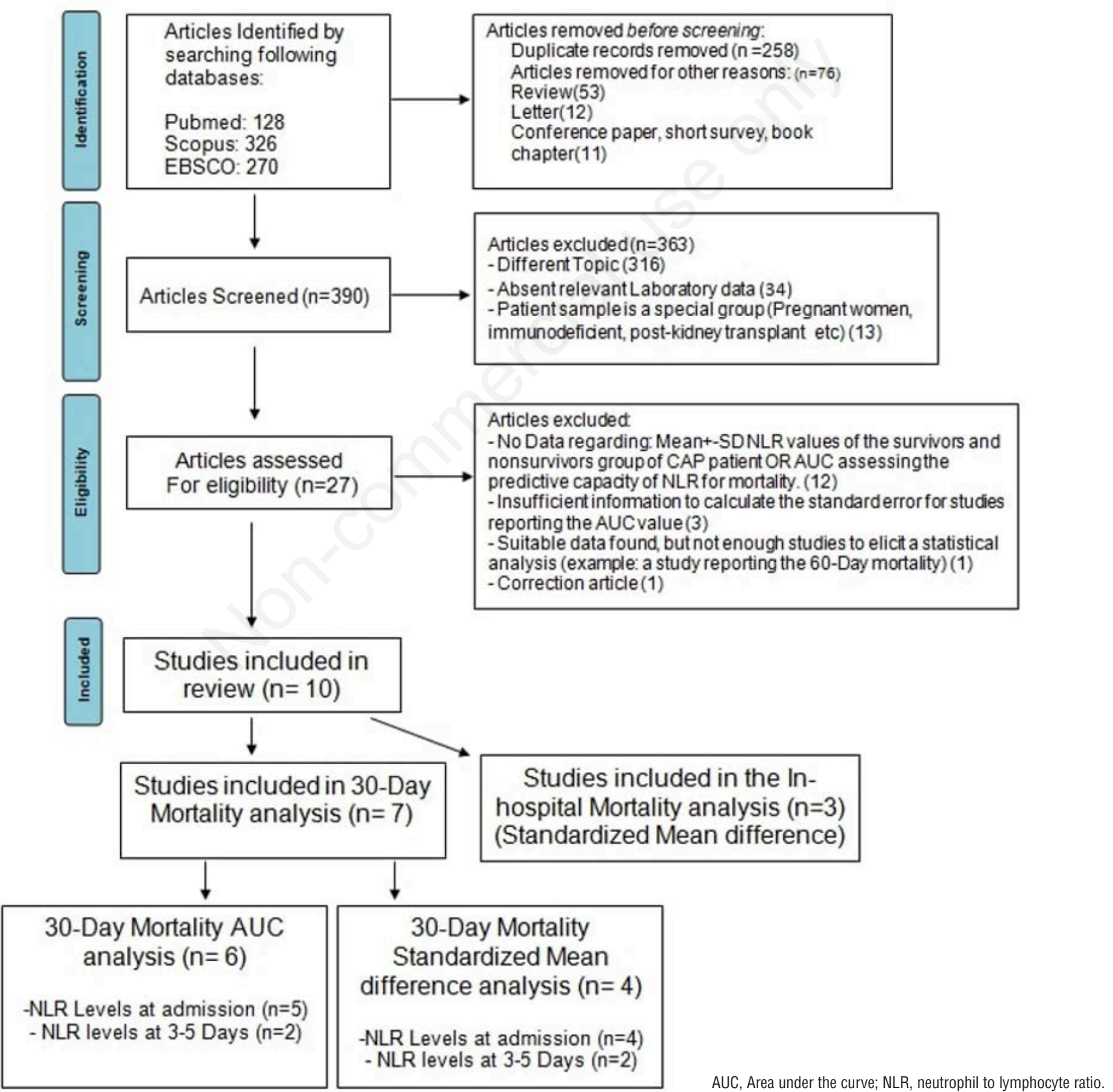

Figure 1. The PRISMA flowchart of literature search and study selection. 


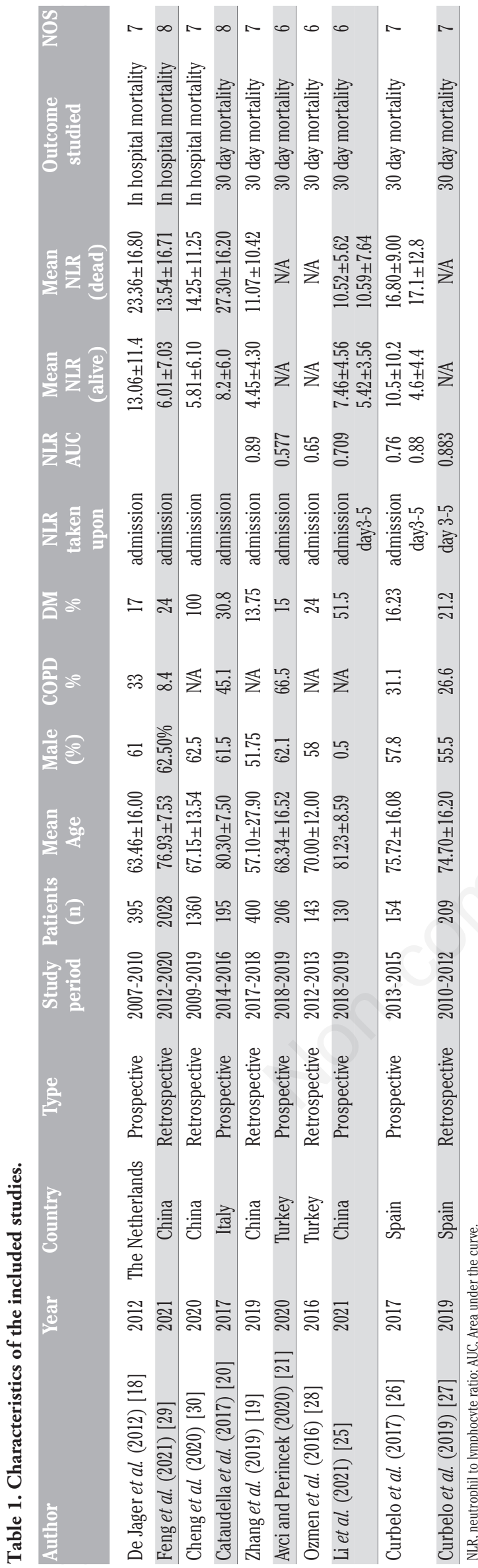

$=66.43 \%$ ). Publication bias and heterogeneity assessment was not done for analysis of NLR levels taken at 3-5 as each analysis only consisted of two studies.

\section{Meta-regression analysis}

We conducted a meta-regression analysis to further assess the effect of confounding factors on SMD between survivors and nonsurvivors. The outcome variable was the SMD of the admission NLR, and the covariates were differences in age (years) and the differences in proportion of male patients (\%) between the non-survivors and survivors of among the 30-day mortality studies. Both the difference in age and differences in proportion of male patients did not have a statistically significant effect, however the differences in proportion of males had a clear positive correlation with SMD of NLR values $(\mathrm{r}=0.76 \mathrm{p}=0.448 ; \mathrm{r}=0.97 \mathrm{p}=0.168$ respectively $)$ (Supplementary File).

\section{Sensitivity analysis}

Stability of the results was evaluated through sensitivity analysis. The corresponding pooled NLR admission SMD values were not substantially altered when single studies were sequentially removed,

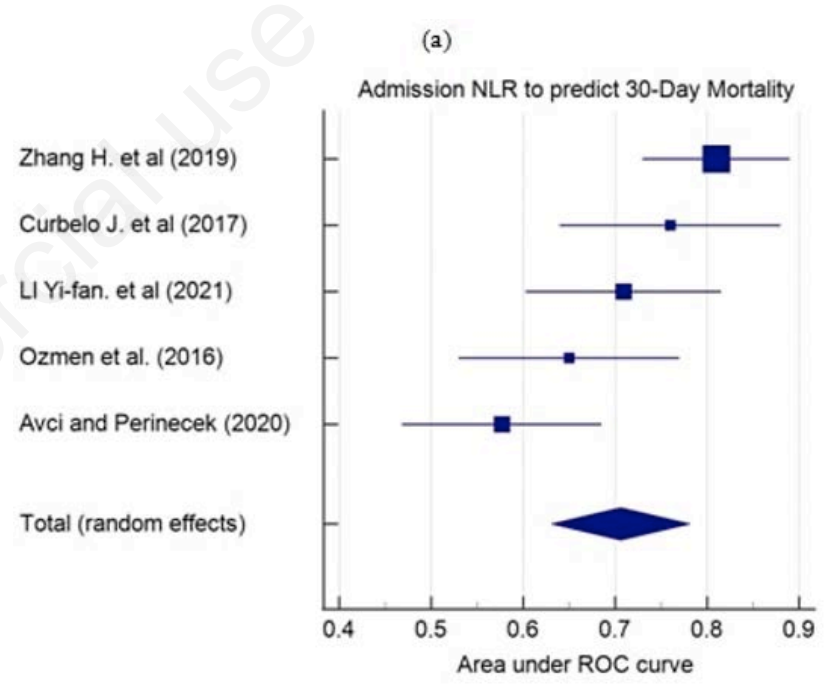

(b)

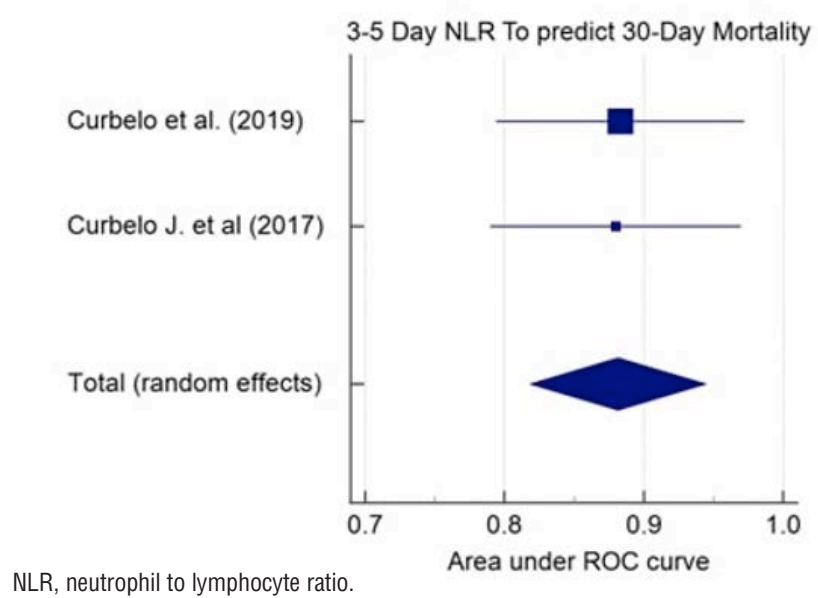

Figure 2. Forest plot of the AUC meta-analysis. a) NLR at admission to predict 30-day mortality. b) NLR at 3-5 days to predict 30-day mortality. 
with an effect size ranging between (0.867 and 1.314) regarding 30day mortality studies and ranging between $(0.936$ and 1.112$)$ regarding in-hospital mortality studies, suggesting that the results of the meta-analysis were stable (Supplementary File). Also, the corresponding pooled AUC was not substantially altered when single studies were sequentially removed, with an effect size ranging between $(0.672$ and 0.743$)$ suggesting that the results of the AUC meta-analysis were stable. (Supplementary File). Meta-regression and sensitivity analysis was not feasible for analysis of NLR levels taken at 3-5 days.

\section{Discussion}

\section{Implications}

The use of the NLR in predicting mortality in patients with CAP has been widely studied, yet the results are inconclusive. To the best of our knowledge, this is the first meta-analysis to determine the role of the NLR in the assessment of mortality in CAP patients.

In this study the NLR of 30-day non-survivors and in-hospital non-survivors was significantly higher than the NLR of survivors among CAP patients. The pooled area under the curve (AUC) representing the ability of admission NLR to predict 30-Day mortality was 0.706 . This value is considered to be significant with an acceptable discriminative ability [31]. However, the pooled area under the curve (AUC) representing the ability of NLR levels taken at 3-5 days to predict 30-day mortality was 0.882 . This value is considered to be significant with an excellent discriminative ability [31].

Curbelo et al. [26], Li et al. [25], and Lee et al. [22] all have illustrated how NLR values taken at early-stage evolution (between 3-5 days) were more accurate than NLR admission levels in predicting 30-day mortality and the difference of mean NLR levels between the survivors and non-survivors group was more significant when NLR levels were taken after 3-5 days from (a)

Cataudella E. et al (2017)

Curbelo J. et al (2017)

LI Yi-fan. et al (2021)

Zhang $\mathrm{H}$. et al (2019)

Total (random effects)

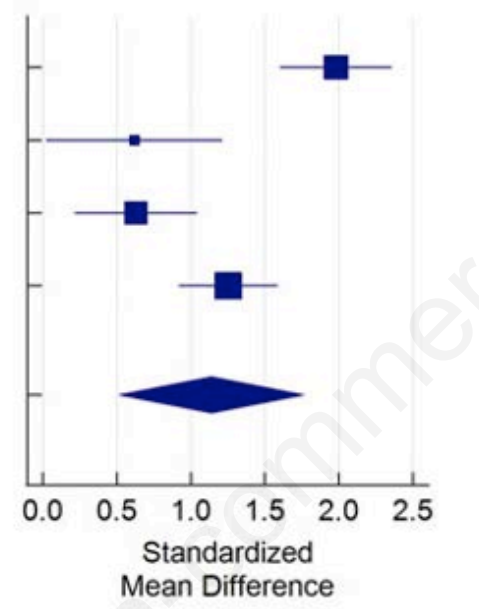

(b)

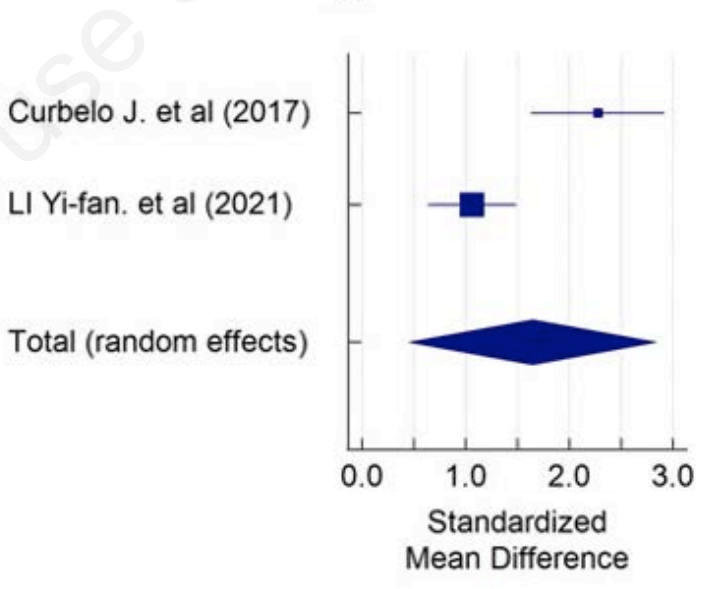

(c)

Cheng et al. (2020)

De Jager et al. (2012)

Feng et al. (2021)

Total (random effects)

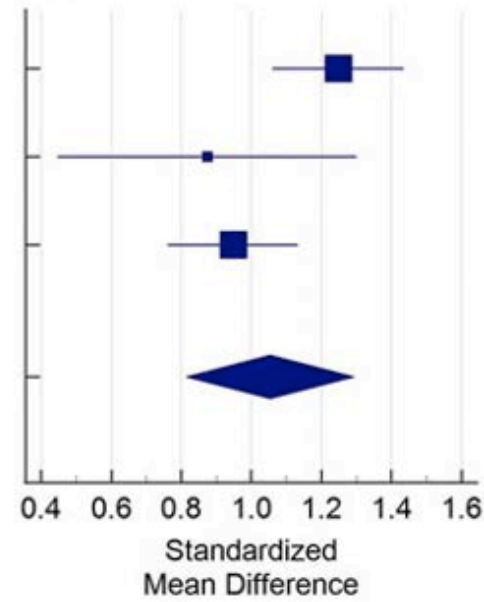

Figure 3. a) Forest plot of the pooled SMD between NLR in the survivors and 30-day non-survivors when NLR levels taken at admission. b) Forest plot of the pooled SMD between NLR in the survivors and 30-day non-survivors when NLR levels taken at 3-5 days. c) Forest plot of the pooled SMD between NLR in the survivors and in-hospital nonsurvivors when NLR taken at admission. 
admission, however both were significant. This correlates with our findings as the pooled AUC and SMD values, when NLR levels were taken between 3-5 days, were higher than the pooled AUC and SMD when admission NLR were used; however, both were significant. This observation can be explained by looking at the data, NLR levels of survivors tend to decrease after 3-5 days, while NLR levels of non-survivors tend to either persist at the same elevated state or further increase. This could reflect a state of uncontrolled immunological response and an ongoing systemic inflammatory process [26]. Keeping in mind the availability and cost effectiveness of NLR, our pooled findings suggest the serious incorporation of NLR into the routine assessment of CAP patients, especially in the process of risk stratification.

Incorporation of NLR could be through making it a part of the initial stratification process of CAP patients; this was suggested by many studies including Lee et al. [22] and Zhang et al. [19] who combined NLR incremental change and NLR levels respectively with pneumonia severity index to obtain a more accurate prediction of 30-day mortality. Another approach suggested by Curbelo et al. [27] is the usage of NLR in the post-acute phase after the start of antibiotic management. CURB-65 and PSI are the conventional scoring methods for pneumonia severity screening. However, they are not without their limitations. CURB-65, while easy to use, is neither sensitive nor specific in predicting mortality and PSI, while more accurate, is made of 20 variables and is not always clinically applicable [19,32].

\section{Limitations}

There were several limitations in this study. Firstly, the small number of studies included. This did not allow for assessing more covariates in the meta regression and did not allow for deeper sensitivity analysis. However, regardless of this limitation, we believe that the main objective of assessing the role of NLR in prediction of mortality among CAP patients was fulfilled. The small number of studies is mainly due to limited number of studies within our objective. Secondly, many studies which have been assessed for eligibility by full text reading, did not report enough data to be accurately included in the analysis. For example, the study by Lee et al. [22] reported the NLR levels taken at 3-5 days as median (IQR), and after checking the skewness it was inappropriate to include this study. This study has illustrated how NLR admission levels tend to decrease in the survivors group and tend to persist in the non-survivors group, and would have supported our pooled findings if it was included. Likewise, some studies did not report enough data to calculate the standard error for the AUC value [22]. (a)

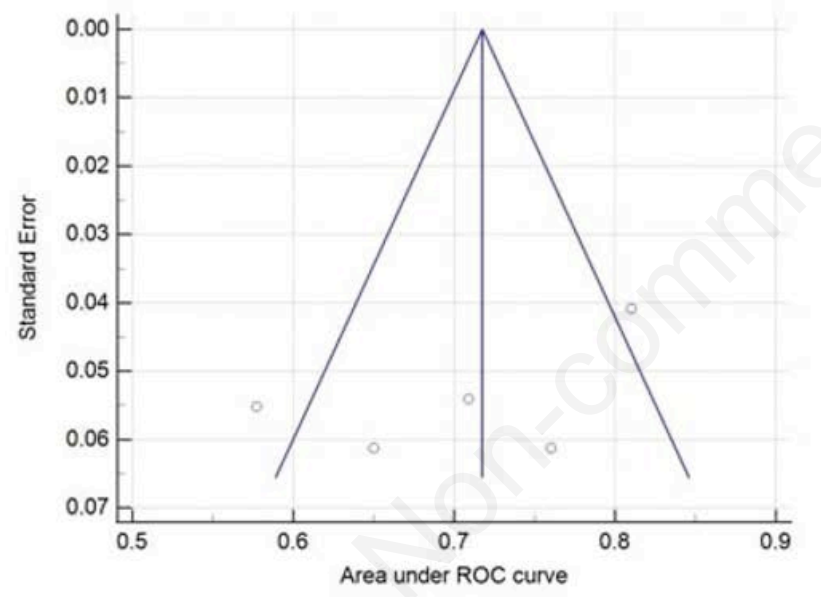

(b)

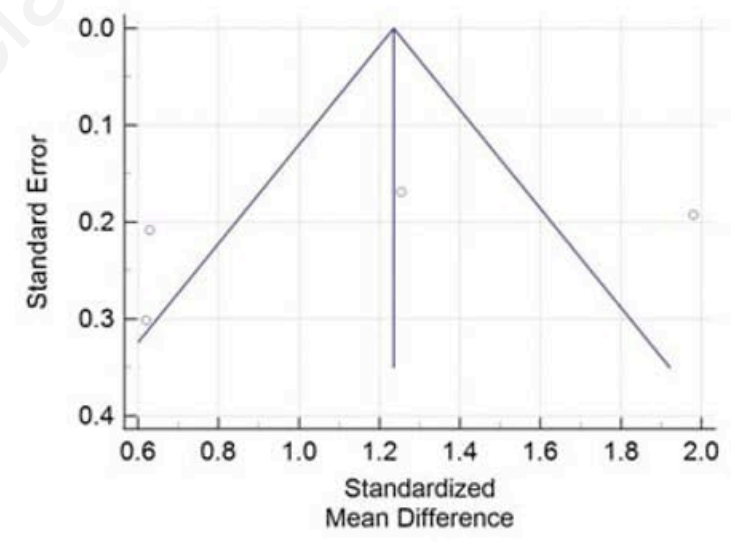

(c)

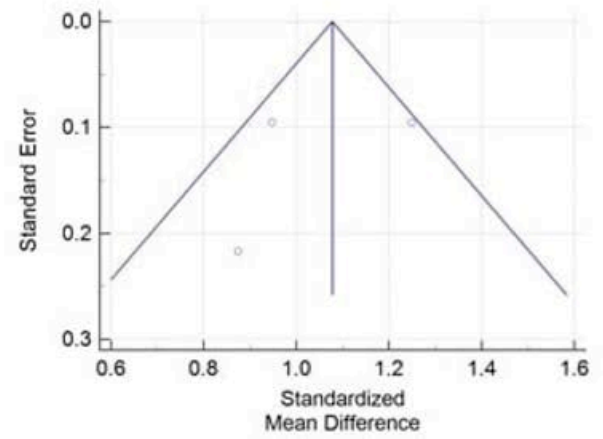

Figure 4. Funnel plots for publication bias assessment. a) Funnel plot of the analysis of admission NLR predictive value and 30-day mortality (pooled AUC). b) Funnel plot of the analysis of differences between the mean admission NLR of the survivors and 30-day non-survivors. c) Funnel plot of the analysis of differences between the mean admission NLR of the survivors and in-hospital non-survivors. 
Thirdly, among included studies, some have only included elderly patients. However, the effect of age upon our analysis was not significant when meta-regression was done.

\section{Conclusions}

NLR is considered a significant predictor of 30-day mortality, especially in the early-stage evolution. Also, NLR is a significant predictor of in-hospital mortality. NLR has a potential to be incorporated in the routine assessment of CAP patients, keeping in mind the availability and cost effectiveness of this test.

\section{References}

1. Wunderink RG, Waterer GW. Clinical practice. Communityacquired pneumonia. N Engl J Med. 2014;370:543-51.

2. Jain S, Self WH, Wunderink RG, et al. Community-acquired pneumonia requiring hospitalization among U.S. adults. N Engl J Med 2015;373:415-27.

3. Pfuntner A, Wier LM, Stocks C. Most frequent conditions in US hospitals, 2011: statistical brief\# 162. 2013;

4. Welte T, Torres A, Nathwani D. Clinical and economic burden of community-acquired pneumonia among adults in Europe. Thorax 2012;67:71.

5. Lim WS. Defining community acquired pneumonia severity on presentation to hospital: an international derivation and validation study. Thorax 2003;58:377-82.

6. Fine MJ, Auble TE, Yealy DM, et al. A prediction rule to identify low-risk patients with community-acquired pneumonia. N Engl J Med 1997;336:243-50.

7. Chalmers JD, Singanayagam A, Akram AR, et al. Severity assessment tools for predicting mortality in hospitalised patients with community-acquired pneumonia. Systematic review and meta-analysis. Thorax 2010;65:878.

8. Yandiola PPE, Capelastegui A, Quintana J, et al. Prospective comparison of severity scores for predicting clinically relevant outcomes for patients hospitalized with community-acquired pneumonia. Ches 2009;135:1572-9.

9. Chalmers JD, Singanayagam A, Hill AT. C-reactive protein is an independent predictor of severity in community-acquired pneumonia. Am J Med 2008;121:219-25.

10. Schuetz P, Suter-Widmer I, Chaudri A, et al. Prognostic value of procalcitonin in community-acquired pneumonia. Eur Respir J 2011;37:384.

11. Kolditz M, Halank M, Schulte-Hubbert B, et al. Copeptin predicts clinical deterioration and persistent instability in community-acquired pneumonia. Respir Med. 2012;106:1320-8.

12. Huang DT, Angus DC, Kellum JA, et al. Midregional proadrenomedullin as a prognostic tool in community-acquired pneumonia. Chest. 2009;136:823-31.

13. Krüger S, Ewig S, Giersdorf S, et al. Cardiovascular and inflammatory biomarkers to predict short-and long-term survival in community-acquired pneumonia: Results from the German Competence Network, CAPNETZ. Am J Respir Crit Care Med 2010;182:1426-34.

14. Kong W, He Y, Bao H, et al. Diagnostic value of neutrophil-lymphocyte ratio for predicting the severity of acute pancreatitis: ameta-analysis. Dis Markers 2020;2020:9731854.

15. Wang Q, Ma J, Jiang Z, Ming L. Prognostic value of neutrophil- to-lymphocyte ratio and platelet-to-lymphocyte ratio in acute pulmonary embolism: a systematic review and meta-analysis. Int Angiol J Int Union Angiol 2017;37:4-11.

16. Ye Z, Ai X, Liao Z, et al. The prognostic values of neutrophil to lymphocyte ratio for outcomes in chronic obstructive pulmonary disease. Medicine (Baltimore) 2019;98:e16371.

17. Rha M-S, Kim C-H, Yoon J-H, Cho H-J. Association between the neutrophil-to-lymphocyte ratio and obstructive sleep apnea: a meta-analysis. Sci Rep 2020;10:10862.

18. de Jager CPC, Wever PC, Gemen EFA, et al. The neutrophillymphocyte count ratio in patients with community-acquired pneumonia. PLoS One 2012;7:e46561.

19. Zhang HF, Ge YL, Wang HY, et al. Neutrophil-to-lymphocyte ratio improves the accuracy and sensitivity of pneumonia severity index in predicting 30-day mortality of CAP patients. Clin Lab 2019;65:190226.

20. Cataudella E, Giraffa CM, Di Marca S, et al. Neutrophil-to-lymphocyte ratio: an emerging marker predicting prognosis in elderly adults with community-acquired pneumonia. J Am Geriatr Soc 2017;65:1796-801.

21. Avci S, Perincek G. The alveolar-arterial gradient, pneumonia severity scores and inflammatory markers to predict 30-day mortality in pneumonia. Am J Emerg Med 2020;38:1796-801.

22. Lee H, Kim I, Kang BH, Um S-J. Prognostic value of serial neutrophil-to-lymphocyte ratio measurements in hospitalized community-acquired pneumonia. PLoS One 2021;16: 0250067.

23. Moher D, Liberati A, Tetzlaff J, et al. Preferred reporting items for systematic reviews and meta-analyses: The PRISMA statement. PLOS Med 2009;6:e1000097.

24. Stang A. Critical evaluation of the Newcastle-Ottawa scale for the assessment of the quality of nonrandomized studies in metaanalyses. Eur J Epidemiol 2010;25:603-5.

25. Li Y-F, Li C-H. Application value of neutrophil to lymphocyte ratio for the elderly patients with community acquired pneumonia in emergency department. Progress in Modern Biomedicine 2021;21:455-8.

26. Curbelo J, Luquero Bueno S, Galván-Román JM, et al. Inflammation biomarkers in blood as mortality predictors in community-acquired pneumonia admitted patients: importance of comparison with neutrophil count percentage or neutrophillymphocyte ratio. PLoS One 2017;12:e0173947.

27. Curbelo J, Rajas O, Arnalich B, et al. Neutrophil count percentage and neutrophil-lymphocyte ratio as prognostic markers in patients hospitalized for community-acquired pneumonia. Arch Bronconeumol 2019;55:472-7.

28. Ozmen I, Karakurt Z, Salturk C, et al. Can N-terminal pro Btype natriuretic peptide, neutrophil-to-lymphocyte ratio, C-reactive protein help to predict short and long term mortality? Bratisl Med J 2017;117:587-94.

29. Feng D-Y, Zou X-L, Zhou Y-Q, et al. Combined neutrophil-tolymphocyte ratio and CURB-65 score as an accurate predictor of mortality for community-acquired pneumonia in the elderly. Int J Gen Med 2021;14:1133-9.

30. Cheng S, Hou G, Liu Z, et al. Risk prediction of in-hospital mortality among patients with type 2 diabetes mellitus and concomitant community-acquired pneumonia. Ann Palliat Med 2020;9:3313-25.

31. Hosmer DW, Lemeshow S. Chapter 5. In: Applied Logistic Regression. 2nd edition. New York: J. Wiley \& Sons; 2000. p. 160-4.

32. Ananda-Rajah MR, Charles PGP, Melvani S, et al. Comparing the pneumonia severity index with CURB-65 in patients admitted with community acquired pneumonia. Scand J Infect Dis 2008;40:293-300. 